\title{
Modeling of GMR loops asymmetry in spin valves
}

\author{
Alexander Kurenkov ${ }^{1,2}$, Georgy Babaizev ${ }^{1,2}$, and Nikolay Chechenin ${ }^{1,2, *}$ \\ ${ }^{1}$ Skobeltsyn Institute of Nuclear Physics, Lomonosov Moscow State University, Leninskie Gory, 1/2, 119991 Moscow, Russia \\ ${ }^{2}$ Faculty of Physics, Lomonosov Moscow State University, Leninskie Gory,1/2, 119991 Moscow, Russia
}

\begin{abstract}
The regular shape of the magnetoresistance as a function of applied magnetic field, $R(H)$, is important for numerous spin valves applications. Nevertheless, in many practical studies the shape of the $R(H)$ curve is unpredictably complicated. A simple technique, proposed in this paper, allows interpreting the $R(H)$ features and calculating $R(H)$ curves from known hysteresis loops of ferromagnetic layers, composing spin valves. And vice versa, the shape of the hysteresis loops of the composing ferromagnetic layers can be obtained from $R(H)$ curves. The method does not give an insight into the physical factors determining the shape of hysteresis loops, but it is suitable for prompt selection of promising spin valve stacks. A series of spin valve structures was prepared and their magnetoresistance curves were measured. The analysis of the experimental data demonstrates the capacity of the proposed method.
\end{abstract}

\section{Introduction}

Spin valves (SV) are widely applied in spintronic devices. Active part of SVs normally consists of ferromagnetic $(\mathrm{F})$ - spacer $(\mathrm{S})$ - ferromagnetic $(\mathrm{F})$ antiferromagnetic (AF). In such structures the magnetization in the first ferromagnetic layer (FF) is free to be influenced by an external magnetic field, while in the magnetization in the second ferromagnetic $(\mathrm{PF})$ is pinned by exchange bias field exerted from the adjacent AF layer. Various applications require SVs with different properties, which means different giant magnetoresistance (GMR) characteristics. Characteristics of linear region of magnetoresistance (MR) as a function of external magnetic field, $H$, like the amplitude of the resistance $R(H)$ and position of the MR peak on the $H$ scale, are of a practical interest. In SV-based magnetic field sensors, the tilt of MR in the linear region defines the precision, and, together with the position of linear region along the $H$ axis, the operating range of the sensor. A high amplitude is desirable for both the high precision and wider operating range, while the tilt compromises between the resolution and the field range. In case of magnetoresistive random-access memory, the switching thresholds are defined by linear region position, while high amplitude is important for sustainable state readout. That is why MR curve shape control and mastering is crucial for development of devices with desirable properties.

The switching field in the MR curves can be estimated by minimizing system's energy in Stoner-Wolfarth model $[1,2]$ or considering macrospin dynamics with Landay-Lifshitz-Gilbert equation $[3,4]$. However, to date, these approaches cannot describe some important features of MR curves, such as hysteresis in the switching field in the increasing and decreasing scans and other additional features of the MR loops, emerging in some cases as illustrated below. Here we propose an estimation method, which interprets MR curve as a consequence of the composition of hysteresis loops of individual $\mathrm{F}$ layers in the SV structures. Method reveals their properties, though, does not provide a deep insight into mechanisms which determine the shape of the hysteresis in the individual layers, like tilt of the PF layer's hysteresis loop, which partly results from ferromagneticantiferromagnetic exchange interaction, and must be a subject of separate considerations.

\section{Sample preparation}

The samples were prepared by DC magnetron sputtering (Ar atmosphere 2,9*10-3 Torr, base pressure $10^{-6}$ Torr) in the presence of permanent magnetic field of about 400 Oe for inducing uniaxial anisotropy in Flayers. The stripe-like structures were deposited through gaps in the mask with lateral dimensions of $200 \times 500 \mu \mathrm{m}$ and with long side of the mask oriented perpendicular to the field. Unidirectional anisotropy appeared due to antiferromagnetic (AF) layer growth on the top of the saturated $F$ layer. Stacks had $\mathrm{Ta}[22] / \mathrm{Ni}_{80} \mathrm{Fe}_{20}[12] / \mathrm{Cu}[3] / \mathrm{Ni}_{80} \mathrm{Fe}_{20}[7] / \mathrm{Ir}_{80} \mathrm{Mn}_{20}\left[\mathrm{t}_{\mathrm{AF}}\right] / \mathrm{T}$ $\mathrm{a}[22]$ structure where the figures in the brackets are the layers thicknesses in $\mathrm{nm}$ with $t_{A F}=1$ to $15 \mathrm{~nm}$. MR curves were measured at room temperature using the four-point contact method with current flowing perpendicular to the swept external magnetic field aligned parallel to the easy axis in the F layers The 
contribution of the anisotropic magnetoresistance is negligible in given setup.

\section{Coupling between magnetization and magnetoresistance}

As a rough approximation, GMR can be described as a function of the angle $\phi$ between magnetization directions in successive ferromagnetic layers [5]:

$$
R_{G M R}=R_{G M R}^{0}(1-\cos (\phi(H))) / 2,
$$

where $R^{0}{ }_{G M R}$ is the amplitude of GMR effect. Dependence $\phi(H)$ can be derived from hysteresis loops assuming macrospin model. Here hysteresis loops describe projections of magnetizations on the axis of external magnetic field and macrospin assumption implies constant magnetization vector length during magnetization reversal. Thus:

$$
\begin{aligned}
& \phi(H)=\arccos \left(m_{F F}(H)\right)-\arccos \left(m_{P F}(H)\right), \\
& R(H)=R^{0}+R^{0}{ }_{G M R}\left(1-\cos \left(\arccos \left(m_{F F}(H)\right)+\right.\right. \\
& \left.\left.\arccos \left(m_{P F}(H)\right)\right)\right) / 2,
\end{aligned}
$$

where $m_{F F}=M_{F F}{ }^{e x t} / M_{s}, m_{P F}=M_{P F}{ }^{\text {ext }} / M_{s}$ are relative magnetizations and $M_{F F}{ }^{\text {ext }}, M_{P F}{ }^{\text {ext }}$ are projections of magnetization vector of FF and PF, respectively, on the direction of the external field.

Eq. (3) was applied to construct MR curves, using predefined FF and PF hysteresis loops, and to fit the experimental MR curves. The fitting parameters were coercivity, $H_{C}{ }^{P F}$, exchange bias, $H_{E B}$, and tilt, $d M / d H$, for PF hysteresis loop (Fig.1a).
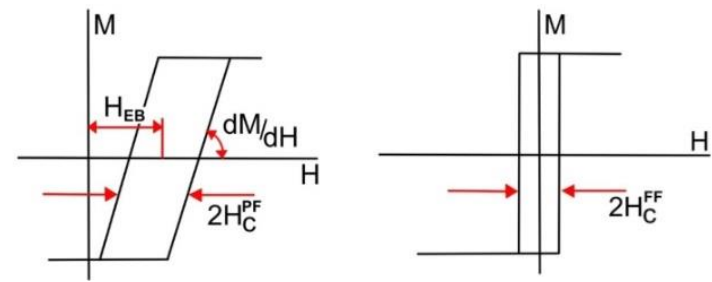

a)

b)

Fig.1 Typical hysteresis loops for pinned (a) and free (b) $\mathrm{F}$ layers and their varied parameters coercivity, $H_{C}{ }^{P F}$, exchange bias, $H_{E B}$, and tilt, $d M / d H$, for PF hysteresis loop (Fig.1a) and solely coercivity, $H_{C}{ }^{F F}$, for the FF hysteresis loops.

Since the magnetic field during the MR measurements was aligned parallel to the easy axis in the samples, the hysteresis loop for FF layer can be approximated by a rectangular shape, while the exchange interaction on the F-AF interface can originate the tilt in the hysteresis loop for PF layer, as schematically illustrated in Fig.1. For the FF hysteresis loops in practical cases in our study, it was found sufficient to vary the coercivity $H_{C}{ }^{F F}$ solely (Fig.1b). If AF had a perfect structure, magnetization reversal of $F$ would occur in the narrow range of external magnetic field, like in the case of $\mathrm{F}$ itself. However, due to variation of $\mathrm{AF}$ grain size and orientation, some of $\mathrm{F}$ domains experience larger exchange bias then the others. This leads to a spreading of magnetization reversal, widening the switching range of the external magnetic field, which manifests in the PF hysteresis loop.

\section{Illustrations of magnetization and magnetoresistance loops coupling}

Fig.2 shows hysteresis loops (a,c,e,g) and MR curves
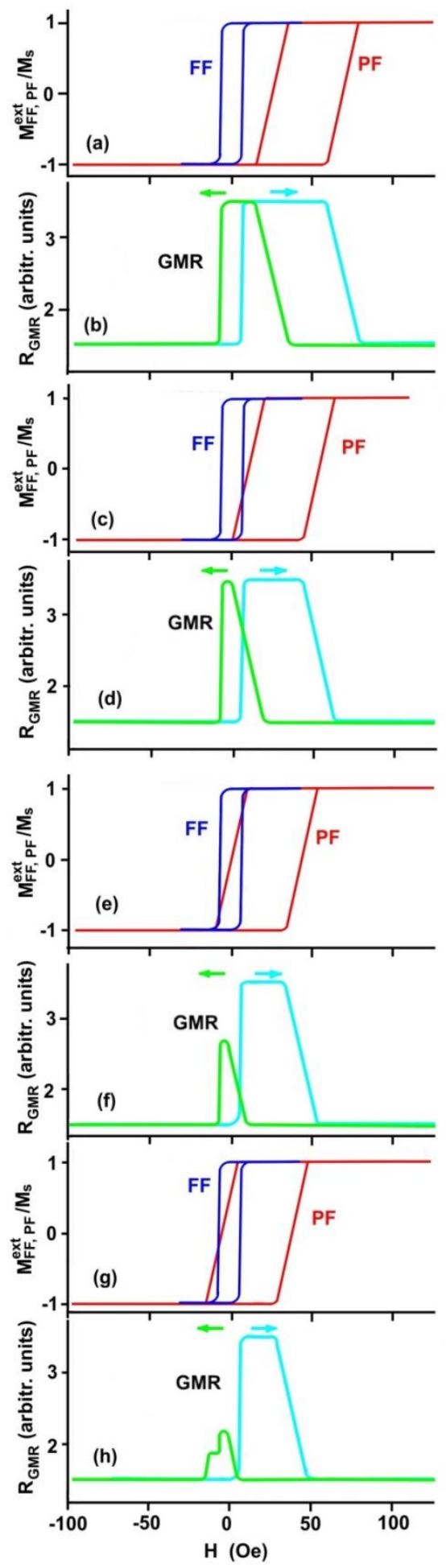

Fig.2 Hysteresis loops (blue - free F, red - pinned F) and corresponding calculated MR curves (light blue for increasing external magnetic field, light-green - for decreasing). Exchange bias decreases from (a) to (h).

(b,d,f,h) for increasing and decreasing external fields, calculated from them. Let us vary solely the exchange bias first (exchange bias decreasing from top to 
bottom in Fig.2). Estimation shows, that when exchange bias, $H_{E B}$, is large enough, compared to coercive field, $H_{c}$, in both FF and PF layers, both forward and backward MR scans (measured with increasing and decreasing external magnetic field) reach GMR effect with a plateau and maximal

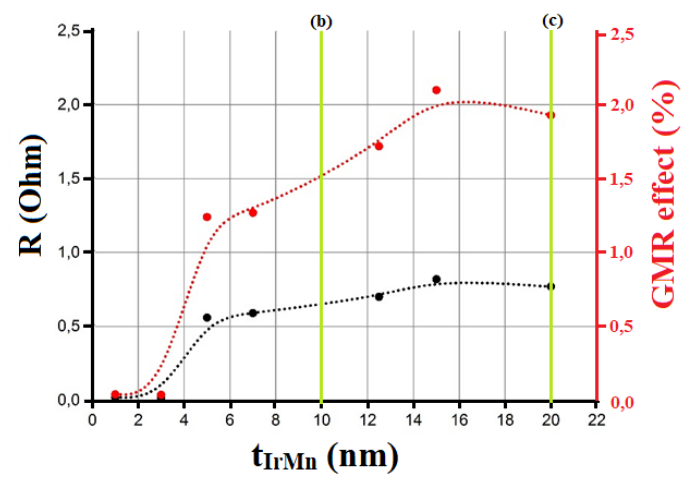

(a)

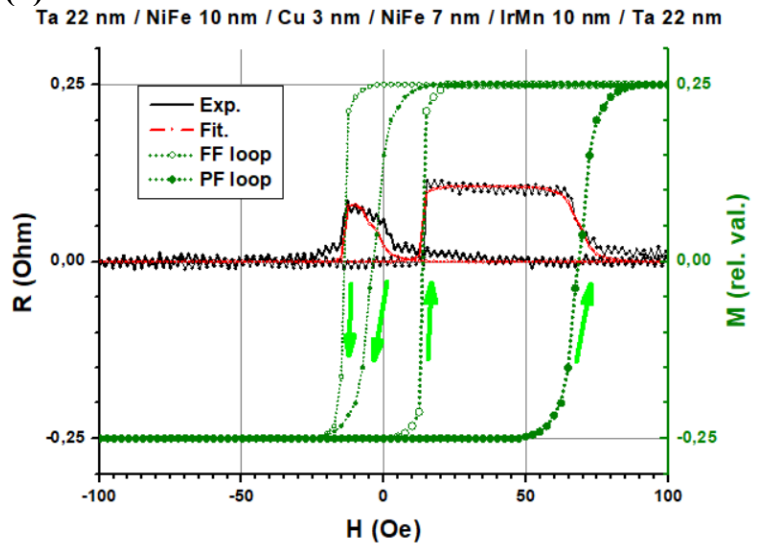

(b)

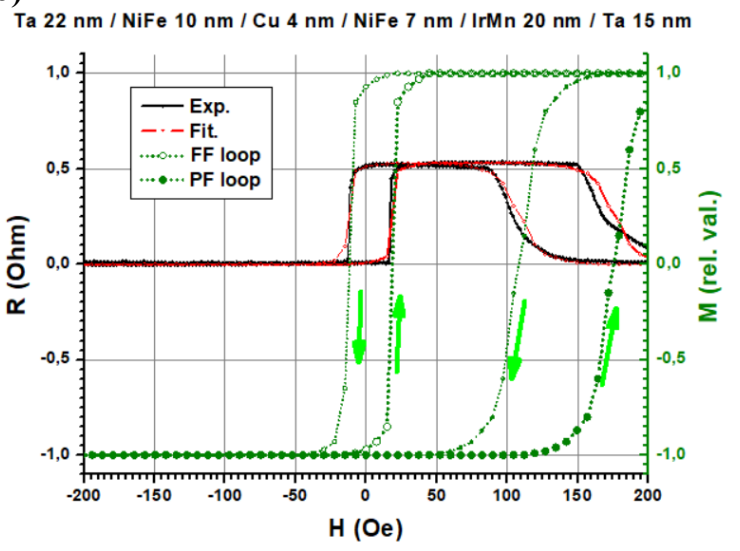

(c)

Fig.3 (a) AF thickness GMR dependence, absolute (black, $\mathrm{Ohm}$ ) and relative (red, percent, the same scale) values. Experimental MR curves (black) with fitting (red) and derived hysteresis loops for FF and PF layers (green) for cases of $10 \mathrm{~nm}-$ (b) and $20 \mathrm{~nm}$-thick (c) AF layer. Insufficient exchange bias (b) leads to superposition of hysteresis loops thus to GMR decrease.

magnitude, Fig. 2a. If exchange bias decreases, the plateaus shorten and, at some point, one of MR curves loses its plateau, Fig. 2d, and then deforms to a narrow form with reduced amplitude, Fig. 2f,h. This evolution of MR curves with a reduction of $H_{E B}$ is experimentally illustrated in Fig. 3. Fig.3a shows experimental dependence of GMR on AF layer thickness. The value of $2 \%$ is rather typical for room temperature for this type of system [6]. Here GMR monotonically increases with the thickness due to increasing exchange bias and saturates at the AF thickness of $15 \mathrm{~nm}$. At $t_{A F}=10 \mathrm{~nm}$, the MR is not at its maximum, Fig. 3a, and the backward (decreasing) field scan shows a lower and narrower MR effect than in the forward (increasing) field scan, Fig.3b. The forward-backward asymmetries in SV structures have been discussed in more details in [7]. Basing on the shapes of the MR scans the FF and PF hysteresis loops can be constructed, as shown in Fig. 3b. The insufficient exchange bias does not allow a complete separation of the FF and PF hysteresis loop causing the reduced (but not zero) GMR. In contrast, the saturated GMR effect at $t_{A F}=20 \mathrm{~nm}$, Fig. 2a, leads to full-magnitude MR curves with plateaus, Fig.3c, evidencing on separated hysteresis FF and PF. By fitting the experimental MR curves the hysteresis loops of composing FM layers can be easily constructed, as shown on Fig.3b,c.

These examples show a critical condition of nonintersecting FF and PF hysteresis loops with large enough exchange bias in PF layer for MR curves with a large amplitude and regular shape. At lower exchange bias, FF and PF hysteresis loops are not separated and intersection causes decrease of GMR. Below, we illustrate that the tilt of the hysteresis loops, $d M / d H$, plays also important role in interpretation of the MR curves shape.

The experimental MR curves, shown in Fig. 4, are of a triangular shape with sharp edge in low-field side, slow decrease at high-field side and asymmetry in the amplitude in the forward-backward scans of the MR measurement. Besides, there is a small additional peak in the negative field region of the backward scan. Fitting of the MR curves with a variation of the FF and $\mathrm{PF}$ hysteresis loops parameters can describe

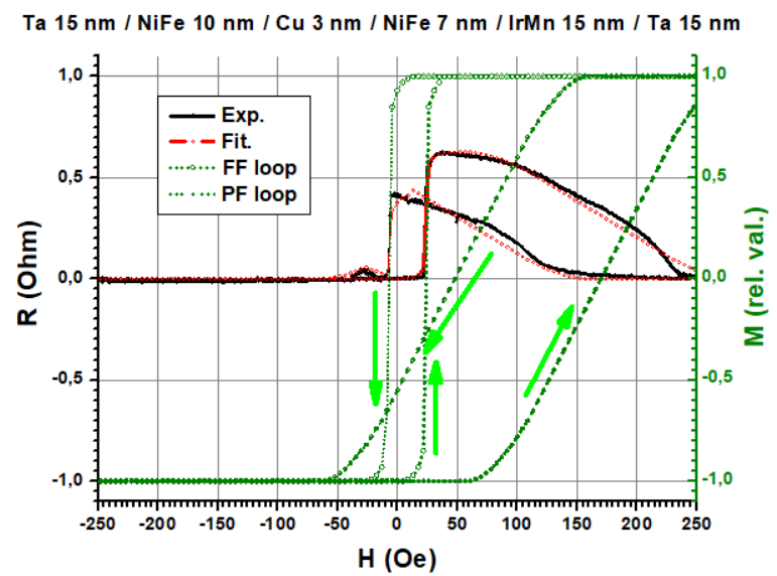

Fig.4 Experimental MR curves (black) with fitting (red) and derived FF and PF hysteresis loops (green). Small extra peak belongs to the MR curve, corresponding to decreasing external magnetic field.

reasonably well the MR curves with their irregular shape at sharp and smooth edges, the asymmetry of amplitude and even the small extra peak. The MR curves features originate from the specific 
combinations of exchange bias, coercivities and tilts of the FF and PF layers. In particular, the additional peak emerges when corresponding hysteresis loops' branches (i.e. both for increasing or both for decreasing field) intersect. From a physical point of layers become equal, reducing to zero the magnetoresistance. At further decrease of the field, the F-layer with smaller anisotropy (smaller tilt of the loop) sharper reverses the magnetization in the rest domains than that in domains in the counterpart Flayer, spiking the MR, as observed in backward scan $R(H)$ around $H \sim-25$ Oe in Fig.4. Evidently, this effect is connected with a local variation of the exchange energy, holding a fraction of the PF domains in the unreversed state and leading to a significant tilt of the $M(H)$ curve. The additional peak can be eliminated not only by a decreasing the tilt, but also by a decreasing the coercive force, or by an increasing exchange bias of PF hysteresis loop.

In practice, each MR curve is a combined effect of a number hysteresis loops' parameters which are not taken into account in our simple approach. Nevertheless, the suggested method, in principle is simple and, at least, can be useful for qualitative interpretation of the MR curves. The method can also be used for inverse task, i.e., to predict the shape of the MR curve, basing on the hysteresis loops of the FF and PF layers. This is very important for optimization of spintronic devices, like spin valves, magnetic field, sensors with required characteristics, reducing the time and resource consuming experimental development.

\section{Conclusions}

A method for analysis of shapes of GMR curves in spin valves of a simple configuration $\mathrm{FF} / \mathrm{S} / \mathrm{PF} / \mathrm{AF}$ is proposed. The method is based on the connection between MR curves and properties of the SV composing ferromagnetic layers. This insight enables constructing SVs with desirable properties by view it means, that magnetization of the FF layer reverses when magnetization of the PF layer still partly keeps the original orientation. Decreasing the external field to the point of intersection, the fractions of domains with reversed magnetizations in FF and PF selecting ferromagnetic layers with proper characteristics. The functionality of the method is illustrated on specific cases of MR measurements. It is proved that it can be helpful in understanding the origin of a series of experimental features in MR curves and can be useful for diagnosing undesirable features of MR characteristics. The method does not give an impact on the nature of the magnetic properties of the components of the SV structure, which make the features of the MR curves, assuming that these issues are to be the subject of further studies of mechanisms and processes, influencing the hysteresis loops in the SV stack components.

\section{References}

1. K. Nishioka, S. Gangopadhyay, H. Fujiwara, M. Parker, IEEE Trans. Magn., 31, 3949 (1995)

2. M.R. Parker, H. Fujiwara, S. Hossain, IEEE Trans. Magn., 31, 2618 (1995)

3. C. Yin, Z. Jia, W.-c. Ma, Y.-1. Ren, Int. J. Miner. Metall. Mater., 20, 700 (2013)

4. J.-S. Park, S.-R. Lee, Y.K. Kim, S.H. Lim, J. Magn. Magn. Mater., 250, 25 (2002)

5. B. Dieny, J. Magn. Magn. Mater., 136, 335 (1994)

6. S. Bae, W. F. Egelhoff, Jr., P. J. Chen, and J. H. Judy, IEEE Trans. Magn. 36(5), 2617 (2000)

7. N. G. Chechenin, P. N. Chernykh, S. A. Dushenko, I. O. Dzhun, A. Y. Goikhman, V. V. Rodionova,. J. Supercond. and Novel Magn, 27, 1547 (2014) 